\title{
TIME TREND ANALYSIS OF RETURN TO WORK AFTER STROKE IN DENMARK 1996-2006
}

\author{
HARALD HANNERZ ${ }^{1}$, OLE S. MORTENSEN ${ }^{3}$, OTTO M. POULSEN ${ }^{1}$, FRANK HUMLE ${ }^{2}$, \\ BETINA HOLBEK PEDERSEN ${ }^{1}$, and LARS L. ANDERSEN ${ }^{1}$ \\ ${ }^{1}$ National Research Centre for the Working Environment, Copenhagen, Denmark \\ ${ }^{2}$ Centre of Rehabilitation of Brain Injury, Copenhagen, Denmark \\ ${ }^{3}$ Bispebjerg University Hospital, Copenhagen, Denmark \\ Department of Occupational and Environmental Medicine
}

\begin{abstract}
Background: In the period 1997-2005, the Danish government initiated a series of legislative changes aimed at facilitating RTW (return to work) in the Danish population. In the present study, we investigated the odds of being gainfully occupied ca. two years after stroke as a function of onset calendar year, 1996-2006. Methods: All previously employed 20-57 year-old stroke patients in Denmark 1996-2006 ( $\mathrm{N}=19985)$ were followed prospectively through national registers. The analysis was controlled for the type of stroke and a series of demographic, structural and occupational variables. Results: The odds for RTW increased significantly during the study period $(\mathrm{P}<0.0001)$. The odds at the end of the period were more than twice as high as they were at the beginning, even after post hoc control for improved survival and decreased unemployment rates. The most conspicuous increase coincided with a change in the sickness benefit act that took place in 2005. Conclusion: The study provides quite strong circumstantial evidence that the legislative changes had an effect on the odds of return to work after stroke. More direct evidence is desirable, but such can only be obtained through a randomized controlled study.
\end{abstract}

Key words:

Cerebrovascular disease, Health policy, Disability retirement scheme, Sickness benefit legislation, Subsidized employment

\section{INTRODUCTION}

The medical profession has traditionally focused on the hospital-based part of stroke rehabilitation, which aims at preventing complications and restoring basic functional skills and mobility [1]. Recovery of social functioning, psychological well-being and life satisfaction can, however, extend years beyond the biomedical rehabilitation period [2]. It is therefore often necessary to supplement hospital treatment with community-based rehabilitation.
Return to work (RTW) is viewed as a key component of such rehabilitation; a stable job and the opportunity to interact with clients or co-workers may enhance self-esteem, which in turn may reduce the risk of secondary disability and substance abuse [3]. The RTW-literature suggests that clinical interventions need to have a tie-in to the workplace and that employer participation, a supportive work climate and cooperation between staff and management are crucial factors in the return to work process [4]. It is also suggested that post-stroke RTW-rates can be significantly

The study was funded by The Danish National Labour Market Authority, Grant Number 2008-5231. The funding source had no involvement in the study design; in the collection, analysis and interpretation of the data; in the writing of the report; or in the decision to submit the paper for publication.

Received: June 23, 2011. Accepted: February 9, 2012.

Address reprint request to H. Hannerz, National Research Centre for the Working Environment, Lersø Parkallé 105, 2100 Copenhagen, Denmark (e-mail: hha@nrcwe.dk). 
influenced by social security systems [1], which, at least in welfare states, are highly dependent on legislation. A recent review points out the importance of promoting legislation which facilitates RTW [3].

Since 1973, disability pensions and sickness absence benefits in Denmark are paid by the municipality, and partially reimbursed by the state [5]. The municipalities play an important role in the return to work process. According to the law, it is not a physician but a municipality official who has the formal right to decide whether or not a person qualifies for sickness benefit, disability pension, or vocational rehabilitation.

In the period 1997-2005, the Danish government initiated a series of legislative changes aimed at improving return to work rates. In 1997, the proportion of the sickness absence benefit that was reimbursed by the state was decreased from $75 \%$ to $50 \%$. In 1998, a flexi-job system was introduced, which allows people with permanently reduced work capacity to work part time, yet through public subsidy get full salary. The municipality paid and the state reimbursed $100 \%$ of the cost. In 1999, the proportion of costs for newly awarded disability pensions that was reimbursed by the state was decreased from $50 \%$ to $35 \%$. In 2001, the flexi-job system was reformed. A person who is eligible to a flexi-job position was entitled to unemployment benefits if no such position could be found. The state reimbursed $65 \%$ of the cost for flexi-job workers and $35 \%$ of the cost for the unemployed. At the same time, reimbursement for vocational rehabilitation was increased from $50 \%$ to $65 \%$. In 2003 , the disability retirement scheme was reformed. A new procedure for assessing work capacity was introduced, and disability pension would only be granted if work ability was permanently reduced and flexi-job work was unfeasible. From July 2005, the municipal control of the sick-listed was intensified by a change in the Sickness Benefit Act. Follow-up evaluations were required once a month instead of once every two months, and a reintegration plan was to be drawn up after four instead of six months. The act also stipulates that the municipality should coordinate its actions with other actors such as employers, labor unions, employment agencies, physicians, hospitals and vocational rehabilitation institutions [5].

In the present study, we investigated the odds of returning to work as a function of the onset calendar year, 1996-2006, among previously employed stroke patients in Denmark.

\section{MATERIALS AND METHODS}

All 20-57 year-old stroke patients in Denmark who had been gainfully occupied prior to a stroke occurring in the period 1996-2006 were included in the study. The patients were followed prospectively through national registers, which contain person-based information about hospital contacts, deaths, migrations and employment.

For any given patient, the calendar year of the stroke was defined as year 0 , the next calendar year was defined as year 1 etc. Multi-level logistic regression was used to model the odds of being gainfully occupied during the major part of calendar year 2, as a function of onset calendar year. Municipalities were treated as the subjects, while individual observations within municipalities were treated as correlated repeated measurements. The analysis was controlled for gender, age, occupational class, self-employment (yes; no), type of stroke, and a variable indicating whether or not a person's municipality had a brain injury rehabilitation center at the time of the stroke.

Further details about the materials and the methods are given in an open access study protocol [6], which was published before we looked at any relation between the exposure and response variables in our data material. The study complies with the Danish Act on Processing of Personal Data (Act No. 429 of 31 May 2000). The data usage was approved by the Danish Data Protection Agency, journal number: 2001-54-0180. 


\section{RESULTS}

The inclusion criteria were fulfilled by 19985 patients and follow-up data was available on 19903 of them. The chance of RTW was highly dependent on onset calendar year $(\mathrm{P}<0.0001)$; the proportion gainfully occupied patients at follow-up ranged from 54\% (956/1783) in 1996 to $72 \%(1300 / 1797)$ in 2006 . With control for covariates, the odds-ratio (OR) between patients hospitalized in calendar year 2006 and those hospitalized in 1996 was 2.40 (95\% CI: 2.07-2.79). OR by calendar year is given in Figure 1 . The distribution of the employment status at follow-up is given by calendar year in Table 1 .

A part of the calendar year effect might be due to improved survival; if more people survive their stroke, then more people have an opportunity to return to work. Another part might be due to a general decrease in unemployment rates. To investigate the influence of these factors, we performed post hoc adjustments of the '2006 vs. 1996' OR. When conditioning on being alive at followup, the OR was 2.50 (95\% CI: 2.11-2.85). When changing the outcome from 'return to work' to 'return to the

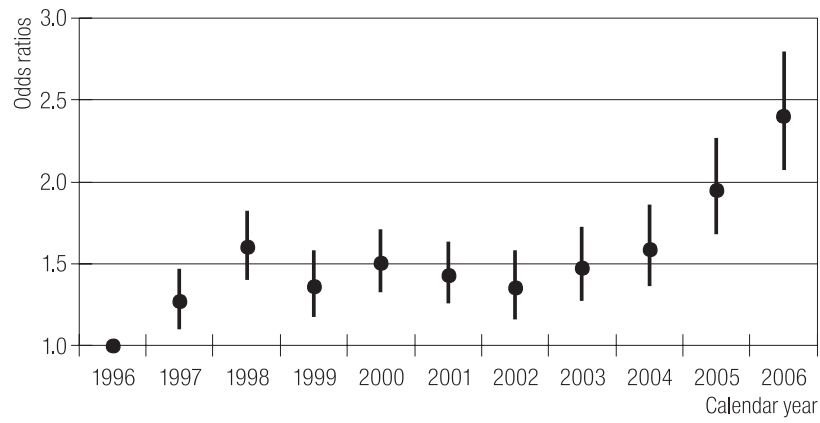

Fig. 1. Odds ratios with $95 \%$ confidence interval for return to gainful occupation two years after stroke, by onset calendar year, among the previously employed stroke patients in Denmark. Calendar year 1996 is the reference year

labor force' (i.e. being gainfully occupied or unemployed vs. being dead, retired or otherwise economically inactive) the OR was 2.11 (95\% CI: 1.85-2.52).

\section{DISCUSSION}

We found that the odds of return to work among previously employed stroke patients increased significantly during the studied time period. The odds at the end of the period

Table 1. Distribution of employment status 2 years after stroke, by onset calendar year, among previously employed stroke patients in Denmark

\begin{tabular}{|c|c|c|c|c|c|c|}
\hline \multirow[b]{2}{*}{ Calendar year } & \multicolumn{5}{|c|}{$\begin{array}{c}\text { Employment status } \\
(\%)\end{array}$} & \multirow[b]{2}{*}{ Total } \\
\hline & gainfully occupied & unemployed & retired & $\begin{array}{c}\text { otherwise } \\
\text { economically } \\
\text { inactive }\end{array}$ & dead & \\
\hline 1996 & 53.6 & 3.1 & 26.5 & 4.1 & 12.7 & 100.0 \\
\hline 1997 & 58.7 & 3.1 & 20.2 & 3.9 & 14.1 & 100.0 \\
\hline 1998 & 63.8 & 3.3 & 16.9 & 4.2 & 11.8 & 100.0 \\
\hline 1999 & 60.8 & 3.5 & 20.5 & 5.0 & 10.2 & 100.0 \\
\hline 2000 & 62.5 & 2.5 & 17.1 & 5.7 & 12.2 & 100.0 \\
\hline 2001 & 60.5 & 3.7 & 18.1 & 6.6 & 11.3 & 100.0 \\
\hline 2002 & 59.7 & 4.0 & 18.7 & 7.7 & 9.9 & 100.0 \\
\hline 2003 & 61.2 & 3.5 & 17.0 & 6.9 & 11.4 & 100.0 \\
\hline 2004 & 63.1 & 2.0 & 17.7 & 7.5 & 9.7 & 100.0 \\
\hline 2005 & 67.9 & 1.6 & 15.5 & 6.5 & 8.5 & 100.0 \\
\hline 2006 & 72.3 & 0.5 & 12.6 & 7.2 & 7.5 & 100.0 \\
\hline
\end{tabular}


were more than twice as high as they were at the beginning, even after post hoc control for improved survival and decreased unemployment rates.

The study is free from sampling bias as all people in the target population were included. Referral bias should be minimal since all of the concerned diagnoses require hospital treatment. The study is also free from recall bias and non-response bias since it was based on national registers, which contained follow-up data on all, but negligibly few (0.4\%) who emigrated during the two-year follow-up period. The high statistical power enables us to conclude not only that the time effect as a whole was statistically significant, but also that the odds for RTW in each of the studied calendar years were significantly higher than they were in 1996, the reference year. We can moreover conclude that the odds in 2006 were significantly higher than they were in each calendar year in the time period 1996-2004. Diagnostic practices might have changed with time. We cannot therefore rule out the possibility that the trend has been influenced by detection bias. However, the number of patients enrolled in 2006 did not differ from the number of patients enrolled in 1996. Another weakness is that the design of the study does not allow us to isolate the effects of the legislative changes. The trend reflects the combined effects of changes in legislation, medical care, employment opportunities, and life style. We were able to discount the effects of decreased unemployment rates and improved survival, but we were not able to control for other possible effects of changes in medical care and we have not been able to control for life style. The smoking prevalence in Denmark decreased gradually throughout the study period, while the prevalence of obesity and excessive drinking increased [7]. These lifestyle changes may have influenced the trend, but since they were of a continuous and gradual nature they cannot explain the sudden increase in RTW rates that took place in 2005-2006. The same holds for changes in medical care. The proportions of stroke patients who received early antiplatelet therapy, early anticoagulant therapy and early computed tomography, increased during the study period, but these changes were also continuous and gradual [8]. Thrombolysis for acute ischemic stroke has been an approved medical therapy in Europe since 2002. The introduction of this therapy can, however, not explain much of the trend in RTW since only about $2 \%$ of all Danish stroke patients were given the therapy in 2006 [9].

\section{CONCLUSION}

We have shown that the odds for RTW after stroke improved substantially during the study period and with regard to the legislative changes, we have provided quite strong circumstantial evidence of an effect, at least since the change in the sickness benefit act that took place on July 1, 2005. More direct evidence is desirable, but such can only be obtained through a randomized controlled trial.

\section{REFERENCES}

1. Treger I, Shames J, Giaquinto S, Ring H. Return to work in stroke patients. Disabil Rehabil 2007;29:1397-403.

2. Wolfenden B, Grace M. Returning to work after stroke: a review. Int J Rehabil Res 2009;32:93-7.

3. Shames J, Treger I, Ring H, Giaquinto S. Return to work following traumatic brain injury: trends and challenges. Disabil Rehabil 2007;29:1387-95.

4. Williams RM, Westmorland M. Perspectives on workplace disability management: a review of the literature. Work 2002;19:8793.

5. Johansen K, Andersen JS, Mikkelsen S, Pass O, Raffnsøe S, Lynge E. Controlling sickness absence: a study of changes in the Danish sickness absence legislation since 1973. Health Policy 2008;86:109-18.

6. Hannerz H, Pedersen BH, Poulsen OM, Humle F, Andersen LL. Study protocol to a nationwide prospective cohort study on return to gainful occupation after stroke in Denmark 1996-2006. BMC Publ Health 2010;10:623. 
7. Ekholm O, Kjøller M, Davidsen M, Hesse U, Eriksen L, Christensen AI, et al. Sickness and health in Denmark \& development since 1987. København: Statens Institut for Folkesundhed, 2006 [in Danish].

8. Sundhed.dk. Quality of stroke treatment [cited 11 January 2011]. Available from URL: https://www.sundhed.dk/ borger/valg-af-laege-og-sygehus/kvalitet-i-behandlingen/ maalinger-af-kvalitet-beh-nip/apopleksi-nip/.

9. Modrau B, Vestergaard K, Iversen HK, Homburg AM, Boysen G, Andersen G. Thrombolysis for acute ischaemic stroke. Ugeskr Laeger 2007;169:3383-5 [in Danish].

This work is available in Open Access model and licensed under a Creative Commons Attribution-NonCommercial 3.0 Poland License - http://creativecommons.org/ licenses/by-nc/3.0/pl/deed.en. 